\title{
Counter a Drone and the Performance Analysis of Deep Reinforcement Learning Method and Human Pilot
}

\author{
Ender Çetin, Cristina Barrado, Enric Pastor \\ Technical University of Catalonia (UPC BarcelonaTech) \\ Computer Architecture Department \\ Castelldefels, Spain \\ ender.cetin@upc.edu
}

\begin{abstract}
Artificial Intelligence (AI) has been used in different research areas in aerospace to create an intelligent system. Especially, an unmanned aerial vehicle (UAV), known as a drone, can be controlled by AI methods such as deep reinforcement learning (DRL) in different purposes. Drones with DRL become more intelligent and eventually they can be fully autonomous. In this paper, DRL method supported by real time object detection model is proposed to detect and catch a drone. Additionally, the results are analyzed by comparing the time to catch the target drone in seconds between DRL method, human pilot and an algorithm which directs the drone towards the target position without using any AI method or navigation and guidance method. The main idea is to catch a drone in an environment as fast as possible without crashing any obstacles inside the environment. In DRL method, the agent is a quadcopter drone and it is rewarded in each time step by the environment provided by Airsim flight simulator. Drone is trained to catch the target drone by using DRL model which is based on deep Q-Network algorithm. After training, the tests have been made by the agent drone with DRL model and human pilots to catch stationary and non-stationary target drone. The training and test results show that the agent drone learns to catch target drone which can be a stationary and a non-stationary. In addition. the agent avoids crashing any obstacles in the environment with a minimum success rate of 94\%. Also, DRL model performance is compared with the human pilot performances and the agent with DRL model shows better time to catch the target drone. Human pilots struggle to control the drone by using remote controller when catching the target in simulation. However, the agent with DRL model is rarely missing the target when trying to catch the target.

Index Terms-Counter-Drone, UAV, Drones, AI, Deep Reinforcement Learning, DDQN, Airsim, Image Processing
\end{abstract}

\section{INTRODUCTION}

Recently, the demand for drones have increased tremendously and they have been widely used in many purposes. Additionally, Artificial intelligence (AI) has been also implemented in unmanned air vehicles (UAV) in different purposes. For instance, drones use AI to navigate in an unknown environment by detecting and avoiding the obstacles.

Recently, researchers have proposed some studies about autonomous drones in the area of deep reinforcement learning (DRL). Anwar et al. [1] studied the DRL for autonomous navigation. Transfer learning is applied to reduce the training computation load. The environment is designed in Unreal
Engine [2] and tested in the real world, by using a lowcost drone, with the similar results obtained. However, there are also threats to public security and privacy because of unauthorized, malicious drones. To detect and track these malicious drones, the researchers have presented counterdrone solutions by using different methods and tools including drones equipped with AI used to eliminate UAVs.

Counter drone systems can be categorized as detecting and identifying of an UAV and elimination. Different kind of methods and tools have been investigated by the researchers to localize and track the target drones. For example radar devices can be used to detect and track the drones. Also, acoustic sensors can be utilized to detect, classify and localize drones by using the sounds generated by the motors and propellers. Moreover, object detection algorithms can be implemented to detect drones by processing the images provided by the camera sensors. To eliminate unwanted drones, active counter drone actions are introduced. For example, laser gun, water projector, laser, net, etc. are used to eliminate malicious drones [3].

Shin et al. [4] proposed the position tracking system with multiple Bluetooth receivers and the tracking algorithms. The proposed system tracks the target by estimating the targets location which consists of the distance and angle while using the received signal strength indication signals at four Bluetooth low energy receivers. Moreover, detecting and tracking a malicious drone in a region where Global Positioning System (GPS) is not available can be challenging. Wyder et al. [5], presented a UAV platform to detect and counter a small UAV in an indoor environment without GPS information. In a study presented in MDPI Sensors journal [6], DRL architecture is proposed to counter a drone with another drone. In this study, drone is a quadcopter and it can catch the target drone and it can also autonomously avoid all kind of obstacles inside a suburban neighborhood environment. Considering the recent advances in counter-drone technology by using AI, fighting against malicious drones can be very accurate and efficient. AI can engage with the target faster than the other methods based on human intervention by identifying and classifying the target with a high precision. It is also possible to prevent a false interdiction with the targeted object by using an AI. This is an 
important advantage to be used against drones. The remaining of the paper is organized as follows. In section II, the theory of deep reinforcement learning is provided. Section III provides tools and methods and the human pilot specifications including the DRL model including the environment, states, actions and rewards used in DRL algorithm. In section IV, the training of DRL model and test setup for human pilot is explained and the performance analysis of the DRL method and the human pilot is accessed. Finally, in section V, conclusions and the future work is presented.

\section{Problem Statement}

\section{A. Reinforcement Learning}

Reinforcement learning (RL) is a machine learning method based on trail and error experiences. It is similar to what a baby experiences when learning how to walk. Baby falls over and over again but the parents are always there to teach the baby. In RL, agent like a baby makes decision and takes an action by using environment states. Environment can be considered as the parents in the baby example. Environment states are updated by each action the agent takes and environment outputs a reward which is a scalar value. An action can be analyzed by checking rewards whether that action results positive or negative feedback. In this paper, the drone, a quadcopter, is used as an agent and it will be rewarded in each time step by the environment. The objective of the agent is to maximize the cumulative reward.

The interaction between the agent and the environment is shown in Fig. 1. State is represented as $S_{t}$ and the State Space is represented as $S$. The interaction between the agent and the environment is in discrete time steps $t$. Action and Action Space are represented as $A_{t}$ and $A\left(S_{t}\right)$ respectively. Reward values are updated in each time, $R_{t+1}$, and a new state becomes $S_{t+1}$. In RL, states are mapped to probability of the possible actions in each time step $t$ and it is called policy. The policy is chosen to maximize the cumulative reward over time. The fundamental concepts for RL are explained in detail in surveys [7], [8].

\section{B. Deep Reinforcement Learning}

Deep neural networks are used as decision making algorithm in DRL. In a research by Mnih, V. et all, it is shown that DRL algorithms can beat human performance level in video and board games [9] by implementing a Double deep Q-network (DDQN) [10], an extension of the deep Q-network (DQN) implementation [11]. DQN method is published by DeepMind [9] and the main goal of DQN is to use a deep convolutional neural network to approximate the optimal actionvalue function. DQN provides updating action values and target values iteratively. Moreover, experience replay which randomizes the data and improves the data distribution. Experience replay is demonstrated and explained more in researches [12] , [13] and [14].

DDQN method proposed in [15] and it is basically based in decoupling action selection from evaluation. Although one estimate is updated per step, two estimates are learned in a

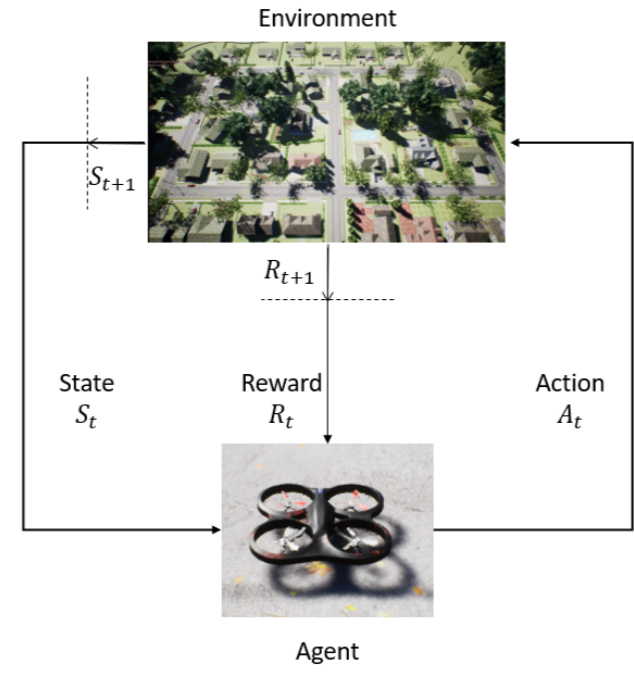

Fig. 1: The agent-environment interaction in reinforcement learning

random selection. The Double DQN and DQN algorithms are explained in detail in a research by Kersandt, K. [16] which uses these algorithms to train and test a drone agent. In this research, DQN and DDQN methods are utilized for training and testing the drone agent.

\section{TOOLS \& Methods}

\section{A. Tools}

In this section the tools available for simulation, AirSim Simulator [18], the toolkit such as OpenAI-Gym [17] for developing and comparing DRL algorithms and state of the art object detection model to detect drones are presented.

1) Airsim Simulator: AirSim is a platform for AI research to experiment with deep learning, computer vision and reinforcement learning algorithms for autonomous vehicles, and it is built on Unreal Engine [2]. Unreal Engine provides ultra realistic rendering and strong graphic features for the Airsim. Airsim has a lot of environments available to be used in reinforcement learning. These environments are mountains, blocks, neighbourhood, city environment etc. In this paper, The neighbourhood environment is selected for training and testing. Environment and Quadcopter used in the Airsim simulator can be seen in Fig. 1. Airsim supports sensors such as camera, Global Positioning System (GPS), LIDAR sensors. AirSim API provides different type of images such as Scene, DepthPlanar, DepthPerspective, DepthVis, DisparityNormalized, Segmentation, SurfaceNormals, and Infrared. In this study, Scene and DepthPerspective image types are used. Additionally, camera sensor on quadcopter provides images in different angles such as front center, front right, front left, bottom center and back center of the quadcopter.

2) OpenAI-Gym: OpenAI-Gym is an open source interface to reinforcement learning tasks. It is a toolkit for developing and comparing reinforcement learning algorithms. It is compatible with TensorFlow [19]. The gym library has a collection 
of environments to test reinforcement learning algorithms. These environments have a shared interface which allows to write general algorithms. Custom environments can also be created by the users to train and test RL algorithms.

3) Drone Detection by State of the Art Object Detection Model - EfficientNet: EfficientNet [20] is a state-of-the-art object detection model and it has become very popular in a short time thanks to its accuracy and efficiency. EfficientNet allows information to flow in different directions: top down and bottom up by its novel Bi-directional Feature Pyramid Network (BiFPN). In addition, EfficientNet uses a fast, normalised fusion technique, which adds an additional weight for each input feature, thus identifying the importance of each input feature. A scaling method which jointly resizes the resolution/depth/width of the model to better fit with different resource constraints is also introduced in EfficientNet..

In this study, state-of-the-art object detection algorithm, EfficientNet-B0, is used to detect drones. EfficientNet-B0 is a version of EfficientNet adapted for small-size objects. A detailed explanation of drone detection model used in this paper is provided in our previous research [21] which proposes a drone detection model trained by using different kinds of images of drones to obtain a more robust drone detector.

\section{B. DRL Model}

In this paper, DDQN algorithm is chosen for DRL training. The best performance of DDQN algorithm have already been presented in previous studies [22], [6]. DRL model have been changed and the improvements are included considering the new image state which is improved by using the state of the art object detection algorithm and fences on the image state, new reward function and drone actions, updated geo-fence locations.

1) States: In this paper, the state of the DRL model is constructed by concatenating an image state and several scalar inputs. The image and the scalar values are processed with a neural network. The image is an input of a convolutional neural network (CNN), followed by a flatten layer and then a concatenation layer joints the flatten output of the CNN with scalar inputs. Neural network model is explained in detail in our previous research [6].

- Image State

The drone continuously captures 256 x 144 pixels size depth image and scene image by using its onboard camera. The scene image seen in Fig. 2a is processed by drone detection model to create bounding boxes when the target drone is detected on the image. The world coordinates of the target drone position are projected into image pixel coordinates by using the quaternions and rotation matrix. Image processing and the drone detection process can be seen in detail in our previous study [21]. Depth image seen in Fig. $2 b$ is used in DRL model for detecting obstacles. After processing the images, bounding box region in depth image is filled with white color and circles like a target in the dart game are created inside the white bounding box region and it is shown in Fig. 2b. The image after detection can be seen in Fig. 2a.

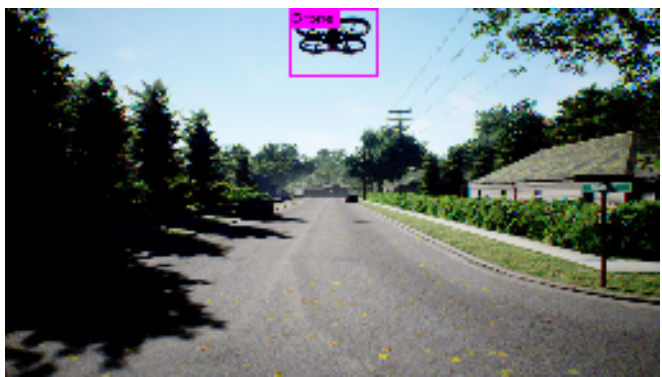

(a) Drone Detection Image

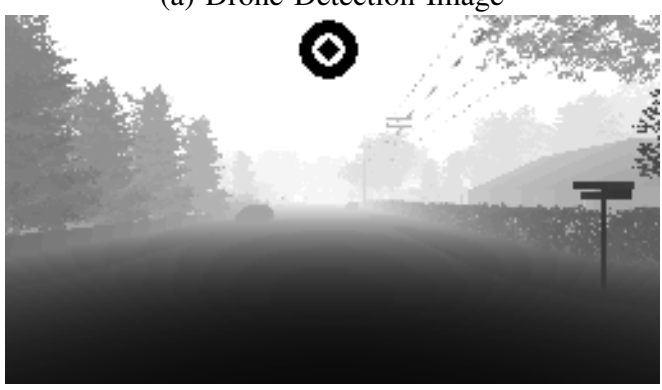

(b) Image After Drone Detection

Fig. 2: Drone Detection and Image Processing

The image state shown in Fig. 3 is set as 30 x 100 pixels which contains $3 \times 10$ pixels black line used to represent the track angle. This line moves left and right while the track angle changes. The image state is the input of a convolutional neural network $(\mathrm{CNN})$ and it is followed by a flatten layer.

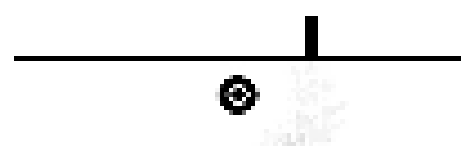

Fig. 3: Image State

In addition, the grid is drawn on the image when the distance between the drone and the geo-fence limits becomes lower or equal to $4 \mathrm{~m}$. The thickness of the grid increases as the drone moves towards the geo-fence limits. An example of the grid image is shown in Fig. 4.

- Scalar Inputs

Scalar inputs contain $v_{x} v_{y}$ agent's velocities in $\mathrm{x}$ and $\mathrm{y}$ directions $d_{x} d_{y} d_{t}$ agent's distances to goal in $\mathrm{x}$ and $\mathrm{y}$ directions and euclidean distance, $d g_{x \min } d g_{x \max } d g_{y \min }$ $d g_{\text {ymax }}$ agent's distances to geo-fence limits, track and elevation angles between the agent and the target. These inputs are received by using APIs provided by the simulation for both the agent and the target drones. In real world, localization and tracking the target drones are not directly available. As it is explained in Section I. There are tools and methods to calculate these angles in real world counter drone systems. 

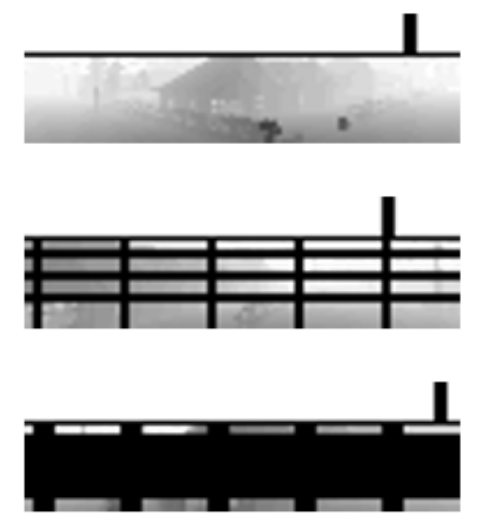

Fig. 4: Fences in Image State

2) Actions: The actions used in DRL contains 3 different options: going forward $4 \mathrm{~m} / \mathrm{s}$, rotate by 30 degree on the left and rotate by 25 degree on the right. However, there is an additional task called attack mode which is added to the actions during testing. If the drone is very close to the target drone, the drone detection model detects a drone and then activates the attack mode to terminate the target. The attack mode is a high speed termination task which is only available in close distances to the target. It is observed that the agent with deep reinforcement learning model moves to the target drone position and reduces the distances to target until the attack mode is activated.

3) Rewards: The reward function is formulated in Table I. Drone detection reward is one of the important factor in this study to achieve better results considering the accuracy of state of the art real time object detection algorithm. The reward function is improved by adding a bounding box information value to the reward calculation. If a drone is detected and the target drone is on the visibility of the agent drone, the agent is rewarded.

TABLE I: Rewards

\begin{tabular}{l|l} 
Reward & The Reason \\
\hline+100 & Target Catched \\
-100 & Collision: Obstacle or geo-fence \\
+1 & Drone Detection \\
$+\zeta$ & Track Angle \\
$-1+\Delta d g$ & Otherwise
\end{tabular}

\section{Performance Analysis \& Results}

In this section, the results of DRL model, human pilots, and the direct solution are discussed. The performance analysis are made by considering the time spent to catch the target and the drone flight parameters recorded during the flight.

\section{A. Training and Test Setup}

In this study, a desktop with NVIDIA GeForce GTX 1060 with 6 GB RAM graphic co-processor and Intel i7 processor,
$16 \mathrm{~GB}$ of memory is used to train the DRL model. Full training with 75,000 steps approximately takes 48 hours. The linear epsilon-greedy policy is adapted and the annealing starts after 25,000 steps. The random factor is kept fixed to $10 \%$ and the Adam optimizer is used in the feed backward of the neural network. DRL method, human pilots and the direct solution are tested with different kind of targets such as stationary target and non-stationary target which moves randomly in a predefined region. In addition, DRL model uses the same resources in testing.

\section{B. DRL Method Training Results}

In Fig. 5a, the training results of DRL method can be seen. The drone is trained with only stationary target. Until the annealing part which starts at 25,000 steps, the drone explore the environment and most of the crashed episodes occur in this part. However, the drone starts catching the target drone in early stages of the training and rewards are going up and down between 100 and -200 . Starting from episode number 500, drone has learned where is the target and reward values are stable around 100. For example, exploration can be identified before episode number 500 in which rewards are very low, around -200 and sometimes they are very close to -300 . At the end of the training, rewards reach even higher values, almost 200. There are still crashed episodes after annealing part because of the random behavior but it is in low level as it is explained earlier in epsilon-greedy policy.

Episode steps are also presented in Fig. 5b to show the details of the time spent on each episode during training. In general, episode steps are higher at the beginning because of exploration but later it becomes stable and less time spent to catch the target.

In Fig.6, it is shown that how frequent each actions are predicted by the agent's neural network to obtain the maximum cumulative reward. The drone spent a lot of time by yawing left or right. It is considered that the higher percentages of these yaw movements are because the agent starts each episodes with random yaw angle.

\section{DRL Method Test Results}

In this section DRL method test results are discussed. After training the drone with DRL method, the drone is tested with different kind of targets such as a stationary drone target or a drone target which moves randomly in the environment. Each test contains 100 episodes.

In Fig. 7a and Fig. 7b, cumulative rewards and time to catch the non-stationary target drone are represented respectively. In Fig. $7 \mathrm{a}$, it is seen that the agent catches the target with a success rate of $96 \%$, but in four episodes which have rewards in between -100 and -150 the agent failed to catch the target and they are penalized. In addition, the time spent during each episodes are generally around 25 time steps in seconds. There are also episodes which take longer time to catch the target. For example, between episodes number 20 and 40, few episodes spent more than 50 time steps to catch the target. The main reason is that the target drone moves randomly and the 


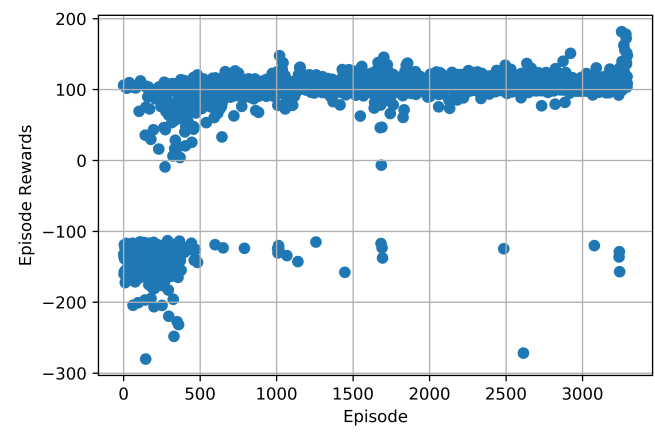

(a) Episode rewards vs Episode number

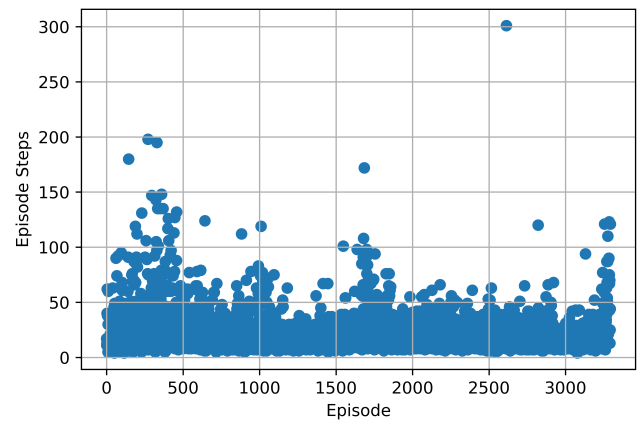

(b) Episode steps vs Episode number

Fig. 5: DRL Method Training Results

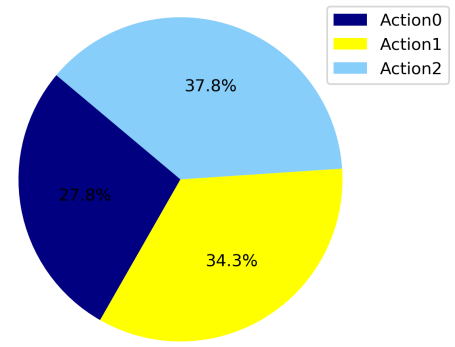

Fig. 6: Action Frequency

agent can miss the target in first try. However, the agent returns back to catch the target, although the agent is not trained to catch a non-stationary drone.

The agent spent less time when the target is a stationary drone. In Fig. 8b, it is shown that the agent spends less than 25 time steps in general to catch the target drone, although some episodes are longer than expected such as 50 time steps. However, it does not mean the agent is failed. On the other hand, the agent successfully catches the target after coming back to face the target drone. The cumulative rewards shown in Fig.8a are around 100 which shows similar trend seen in Fig. 7a except for episode number 54 which has the highest reward value, 114 . The agent catches the target with a success rate of $94 \%$.

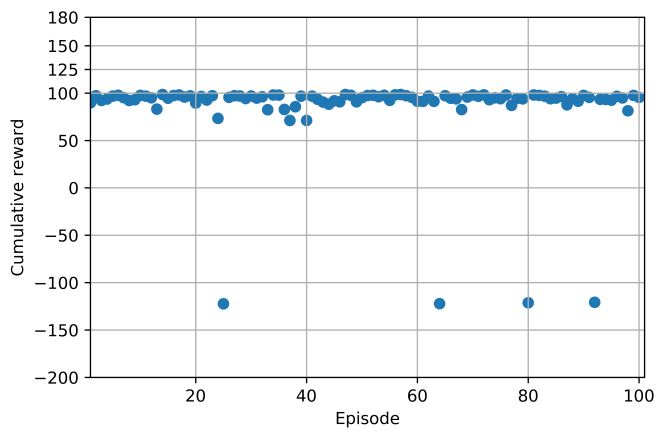

(a) Rewards

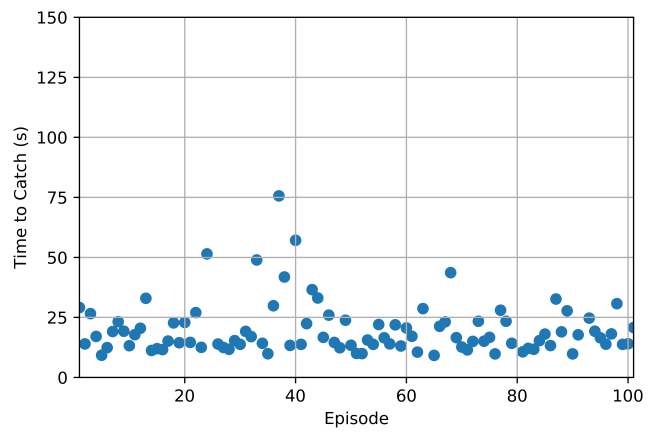

(b) Time to Catch (s)

Fig. 7: DRL Method Test Results for Non-Stationary Target

\section{Direct Solution Method}

In this section, an algorithm directs the drone towards the target position without using any AI method or navigation and guidance method. This is the fastest method and it is considered as an ideal condition since the drone has all the necessary parameters and flight data such as target drone flight attitude angles, target identity, target GPS positions, no obstacles around the target drone.

\section{E. Human Pilot}

Human pilots are performed the same task as the agent did in DRL method to catch the target drone. We have selected two pilots with different skills. One of the pilot has a drone pilot license and the other pilot called "SIM pilot" with more experience on flight simulation but without drone pilot license. Human pilots control the drone by using the remote controller, FrSky Taranis X9D Plus [23]. This is a real UAV remote controller and it can be directly connected to PC via USB port. The pilots with first person view performed the tests to catch stationary drone and non-stationary drone. Licenced drone pilot has trained almost 2 hours in the simulation to catch the target drone. However, the pilots have struggled to control the drone. Most of the problems in test flights are in altitude control of the drone. In real life, human pilots have 


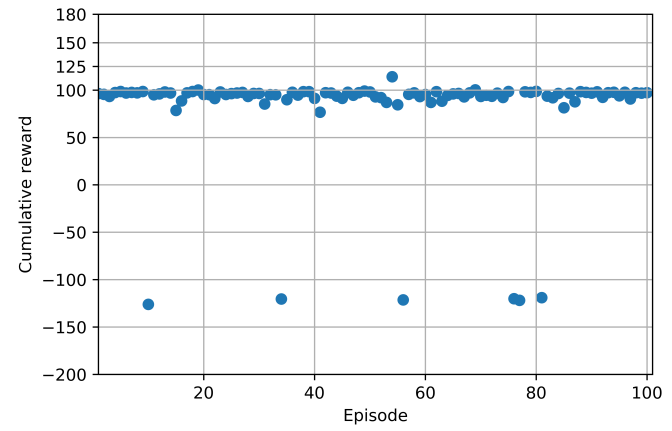

(a) Rewards

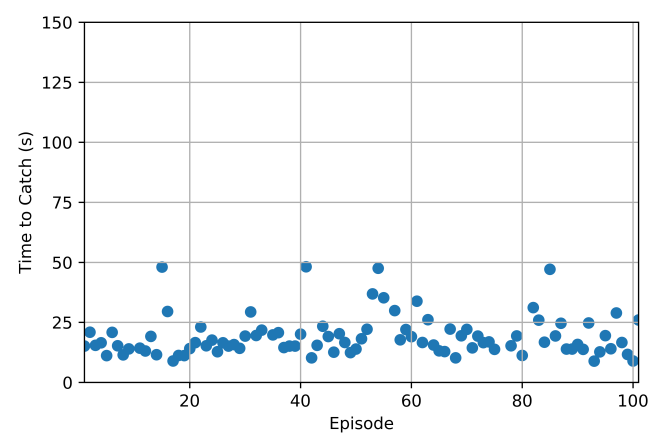

(b) Time to Catch (s)

Fig. 8: DRL Method Test Results for Stationary Target

flight modes such as altitude-hold mode available to them in RC. However, they don't have an access to these modes in the simulation and they need to control the throttle input as well as roll, pitch and yaw control inputs.

To compare human pilot performances with the deep reinforcement learning agents, 10 best episodes are selected for each task. The time spent to catch the stationary target drone are presented in Fig.9 and in Fig.10 for each pilot and DRL agent. In Fig.9 the time spent to catch stationary target by each pilot and DRL agent is presented. Simulation pilot as known as "SIM pilot" is the fastest compared to licenced pilot and the DRL agent. DRL agent spends more time to catch the target because it is not trained to catch the non-stationary target but it can catch it in a longer time than expected average time steps in seconds.

In Fig.10, it is seen that there are big gaps between the whiskers in human pilot performance results compared to AI results. On the other hand, the minimum time to catch a nonstationary drone target or stationary target can be lower in human pilot time results. In other words, human pilots can be faster than DRL method to catch the target drone. However, human pilots are not stable in each test episode. They can miss the target and it can take longer than DRL agent time to come back to catch the target drone. In addition, the median values are represented in horizontal yellow lines in Fig.9 and Fig.10. As it is seen in the figure, human pilot median values out of 10 best episodes are lower than the AI median values.

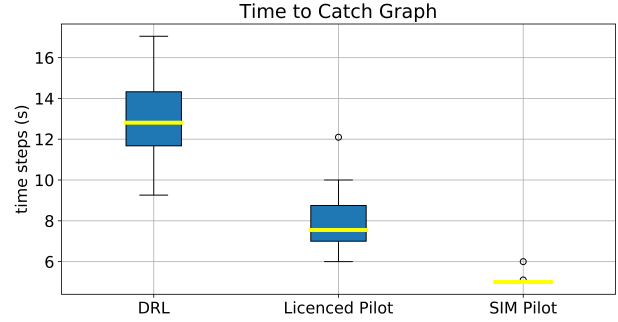

Fig. 9: Results for Non-Stationary Target

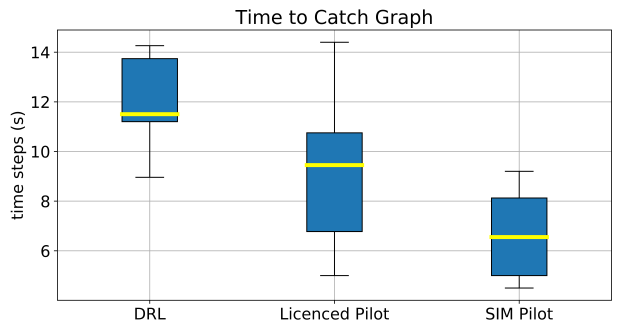

Fig. 10: Results for Stationary Target

Furthermore, the time results for DRL method, human pilots, and direct method are presented in Table II in terms of success rate which accounts for first approach catch, best and worst timing values. Human pilots achieved the best time when catching stationary and non-stationary target. However, human pilots also scored the worst time compared the DRL agent. In Direct solution method explained in Section IV-D, the drone catches the stationary target in 6.1 time steps in seconds. However, when catching non-stationary target, the drone spends 5.1 time steps in seconds, but its worst time is higher, 12.4 time steps in seconds compared to targeting stationary drone which is 6.2 seconds.

\section{TABLE II: Comparison of Results}

\begin{tabular}{l|l|l|l|l|l|l} 
& \multicolumn{3}{|c}{ Stationary Target } & \multicolumn{3}{c}{ Non-Stationary Target } \\
& $\begin{array}{l}\text { Success } \\
(\%)\end{array}$ & $\begin{array}{l}\text { Best } \\
(\mathrm{s})\end{array}$ & $\begin{array}{l}\text { Worst } \\
(\mathrm{s})\end{array}$ & $\begin{array}{l}\text { Success } \\
(\%)\end{array}$ & $\begin{array}{l}\text { Best } \\
(\mathrm{s})\end{array}$ & $\begin{array}{l}\text { Worst } \\
(\mathrm{s})\end{array}$ \\
\hline DRL Agent & 94 & 8.9 & 48.2 & 96 & 9.2 & 75.6 \\
\hline Human Pilots & 100 & 4.5 & 156.5 & 100 & 5.0 & 143.1 \\
\hline Direct Method & 100 & 6.1 & 6.2 & 100 & 5.1 & 12.4
\end{tabular}

\section{Conclusions \& Future Work}

Recent advances in AI can be an efficient method for counter drone systems to fight against unwanted drones. In this research, it is presented that the artificial intelligence method, deep reinforcement learning model can be used to train an agent drone to catch a target drone and the performance of the agent is compared with the human pilot performance. In addition, the agent drone can avoid the obstacles and navigate in the environment. The main contribution of this study is that object detection model output image is processed to be used in the image state in order to increase the efficiency of the 
learning. After that, Image state and several scalar inputs are concatenated.

Response time and the efficiency of tools used to track and catch the drones are very important challenges. In the future, it is considered that the efficiency of the DRL model and the drone detection model can be increased to obtain more robust counter drone system. Drone surveilance technologies such as radar can be utilized to detect and localize drones. Moreover, DRL model can be optimized and trained to catch a target drone which moves in 3D space.

As a future work, DRL agent to counter a drone is considered to be used in public help by local authorities. Trained DRL model will be implemented in a real drone and tested to catch a target drone.

\section{ACKNOWLEDGMENT}

This work was funded partially by the AGAUR under grant 2020PANDE00141, the Ministry of Science and Innovation of Spain under grant PID2020-116377RB-C21 and the SESAR Joint Undertaking (JU) project CORUS-XUAM, under grant SESAR-VLD2 101017682. The JU receives sup- port from the European Union's Horizon 2020 research and innovation programme and the SESAR JU members other than the Union.

Special thanks to Dr. Pablo Royo Chic for his assistance to our paper by piloting a drone to catch the target drone.

\section{REFERENCES}

[1] A. Anwar and A. Raychowdhury, "Autonomous navigation via deep reinforcement learning for resource constraint edge nodes using transfer learning," arXiv preprint arXiv:1910.05547, 2019.

[2] "Unreal engine 4." https://www.unrealengine.com/en-US/what-is-unrealengine-4. Last Accessed: 2019-01-29.

[3] A. H. Michel, Counter-drone systems. Center for the Study of the Drone at Bard College, 2018.

[4] J.-M. Shin, Y.-S. Kim, T.-W. Ban, S. Choi, K.-M. Kang, and J.-Y. Ryu, "Position tracking techniques using multiple receivers for anti-drone systems," Sensors, vol. 21, no. 1, p. 35, 2021.

[5] P. M. Wyder, Y.-S. Chen, A. J. Lasrado, R. J. Pelles, R. Kwiatkowski, E. O. Comas, R. Kennedy, A. Mangla, Z. Huang, X. Hu, et al., "Autonomous drone hunter operating by deep learning and all-onboard computations in gps-denied environments," PloS one, vol. 14, no. 11, 2019.

[6] E. Çetin, C. Barrado, and E. Pastor, "Counter a drone in a complex neighborhood area by deep reinforcement learning," Sensors, vol. 20, no. 8 , p. 2320, 2020.

[7] B. Kiumarsi, K. G. Vamvoudakis, H. Modares, and F. L. Lewis, "Optimal and autonomous control using reinforcement learning: A survey," IEEE transactions on neural networks and learning systems, vol. 29, no. 6 , pp. 2042-2062, 2018.

[8] R. S. Sutton and A. G. Barto, Reinforcement learning: An introduction. MIT press Cambridge, 1998.

[9] V. Mnih, K. Kavukcuoglu, D. Silver, A. A. Rusu, J. Veness, M. G. Bellemare, A. Graves, M. Riedmiller, A. K. Fidjeland, G. Ostrovski, et al., "Human-level control through deep reinforcement learning," Nature, vol. 518, no. 7540, p. 529, 2015.

[10] H. Van Hasselt, A. Guez, and D. Silver, "Deep reinforcement learning with double q-learning.," in $A A A I$, vol. 2, p. 5, Phoenix, AZ, 2016.

[11] V. Mnih, K. Kavukcuoglu, D. Silver, A. Graves, I. Antonoglou, D. Wierstra, and M. A. Riedmiller, "Playing atari with deep reinforcement learning," CoRR, vol. abs/1312.5602, 2013.

[12] M. B. L. . O. R. C. McClelland, J. L., "Why there are complementary learning systems in the hippocampus and neocortex: Insights from the successes and failures of connectionist models of learning and memory," in Psychological Review, 102(3), pp. 419-457, 1995.
[13] M. Riedmiller, "Neural fitted q iteration-first experiences with a data efficient neural reinforcement learning method," in ECML, vol. 3720 , pp. 317-328, Springer, 2005.

[14] L.-J. Lin, Reinforcement Learning for Robots Using Neural Networks. $\mathrm{PhD}$ thesis, Pittsburgh, PA, USA, 1992. UMI Order No. GAX93-22750.

[15] H. V. Hasselt, "Double q-learning," in Advances in Neural Information Processing Systems, pp. 2613-2621, 2010.

[16] K. Kersandt, "Deep reinforcement learning as control method for autonomous uavs," Master's thesis, Universitat Politècnica de Catalunya, 2018.

[17] G. Brockman, V. Cheung, L. Pettersson, J. Schneider, J. Schulman, J. Tang, and W. Zaremba, "Openai gym," 2016.

[18] S. Shah, D. Dey, C. Lovett, and A. Kapoor, "Airsim: High-fidelity visual and physical simulation for autonomous vehicles," in Field and Service Robotics, 2017.

[19] M. A. et al., "TensorFlow: Large-scale machine learning on heterogeneous systems," 2015. Software available from tensorflow.org.

[20] M. Tan and Q. Le, "Efficientnet: Rethinking model scaling for convolutional neural networks," in International Conference on Machine Learning, pp. 6105-6114, PMLR, 2019.

[21] E. Çetin, C. Barrado, and E. Pastor, "Improving real-time drone detection for counter-drone systems," The Aeronautical Journal, pp. 1-26.

[22] E. Çetin, C. Barrado, G. Muñoz, M. Macias, and E. Pastor, "Drone navigation and avoidance of obstacles through deep reinforcement learning," in 2019 IEEE/AIAA 38th Digital Avionics Systems Conference (DASC), pp. 1-7, IEEE, 2019.

[23] “Taranis x9d plus.” https://www.frsky-rc.com/product/taranis-x9d-plus2/. Last Accessed: 2021-06-24. 